\title{
Dependence of Saline-Induced Natriuresis upon Exposure of the Kidney to the Physical Effects
} of Extracellular Fluid Volume Expansion

\author{
John P. Fitzgibbons, F. John Gennari, Howard B. Garfinked, and \\ Stanley Cortell with the technical assistance of Melvin L. Ponte \\ From the Department of Medicine, Tufts University School of Medicine and \\ the Renal Service, New England Medical Center Hospital, \\ Boston, Massachusetts 02111
}

\begin{abstract}
A B S T R ACT In many previous studies, the natriuresis induced by saline loading has been demonstrated to persist even though glomerular filtration rate (GFR) has been decreased to below pre-expansion levels by a reduction in renal artery pressure. In such studies, however, the kidney has been exposed to the effects of volume expansion for varying periods of time before renal artery pressure was controlled. The present experiments were designed to evaluate whether this period of exposure induces critical changes in intrarenal factors that are responsible for the natriuresis.

Experiments were carried out in rats, in which renal artery pressure was decreased to $70 \mathrm{~mm} \mathrm{Hg}$ either at the onset of saline loading (immediate clamping experiments) or after $45 \mathrm{~min}$ of saline loading had elapsed (delayed clamping experiments). In the delayed clamping experiments, consonant with previous studies, mean sodium excretion, $3.2 \mu \mathrm{eq} / \mathrm{min}$, remained markedly increased above control, despite a reduction in GFR to $91 \%$ of the hydropenic control value. In contrast, when renal artery pressure was comparably reduced at the onset of saline loading mean sodium excretion was only trivially increased, $0.4 \mu \mathrm{eq} / \mathrm{min}$, although GFR increased to $140 \%$ of the hydropenic control value.
\end{abstract}

This work was presented in part at the 6th Annual Meeting of the American Society of Nephrology held in Washington, D. C., November 1973.

Dr. Gennari and Dr. Cortell are the recipients of Career Development Awards (HE38,908 and HE35,834) from the Heart and Lung Institute of the National Institutes of Health. Dr. Fitzgibbons' present address is Springfield Hospital Medical Center, Springfield, Mass. Dr. Garfinkel's present address is University of South Carolina Medical Center, Charleston, S. C.

Received for publication 17 June 1974 and in revised form 21 August 1974.
These results exclude an important role for either a circulating hormone or a reduction in plasma oncotic pressure in the natriuretic response to saline loading, and indicate that intrarenal factors are the critical determinants of the natriuresis. We have used the difference in response to saline loading in the immediate and delayed clamping experiments to evaluate the role of two intrarenal factors, interstitial hydrostatic pressure and renal plasma flow. Interstitial pressure changes were estimated from changes in tubular pressure and diameter by using the in situ compliance characteristics of the tubules. In a group of rats saline loaded without aortic clamping, interstitial pressure increased by 4-5 $\mathrm{mm} \mathrm{Hg}$ and renal plasma flow increased by 2.5 $\mathrm{ml} / \mathrm{min}$. During the period of reduced renal artery pressure, however, neither interstitial pressure nor renal plasma flow was detectably increased above control in either the immediate or the delayed clamping experiments.

The only noteworthy difference between the experiments in which a natriuresis occurred (unclamped and delayed clamping studies) and the experiments in which no natriuresis occurred is that in the former group the kidney was at least transiently exposed both to an increase in renal plasma flow and interstitial pressure. These findings indicate, first, that extracellular fluid volume expansion can induce a natriuresis only if the kidney has been exposed to at least a transient increase in either interstitial hydrostatic pressure or renal plasma flow (or both); and, second, that a sustained increase in interstitial pressure and renal plasma flow is not required for the natriuresis to persist.

\section{INTRODUCTION}

Over a decade ago, de Wardener, Mills, Clapham, and Hayter (1) demonstrated that the natriuresis in- 
duced by saline loading persists despite a reduction in renal artery pressure sufficient to reduce the glomerular filtration rate $(\mathrm{GFR})^{1}$ to below pre-expansion levels. Since a reduction in renal perfusion pressure not only reduced GFR, but also reversed the effects of volume expansion on such intrarenal factors as blood flow, and presumably, intrarenal hydrostatic pressures, attention was naturally focused upon extrarenal factors such as plasma oncotic pressure and circulating hormones to account for the persistent diuresis $(1,2)$. It is noteworthy, however, that in these studies and other confirmatory studies $(3,4)$, renal artery pressure was either reduced gradually or was reduced only after a period of saline loading had elapsed. No consideration has been given to the possibility that the transient period of exposure of the kidney to the effects of extracellular fluid (ECF) volume expansion in these studies might have produced crucial intrarenal changes which were not immediately reversed by the subsequent reduction in renal artery pressure. The present studies were therefore undertaken to determine whether prevention of such exposure by a prompt partial reduction in renal artery pressure at the onset of saline loading would alter the natriuretic response.

The results indicate that a period of exposure of the kidney to the effects of volume expansion at a normal perfusion pressure is indeed a prerequisite to the natriuresis. When renal artery perfusion pressure was reduced to $70 \mathrm{~mm} \mathrm{Hg}$ at the very onset of saline loading, no diuresis occurred despite an increase in GFR. Reduction of renal artery perfusion pressure to an identical level after a period of saline loading, on the other hand, failed to abolish the diuresis despite a fall in GFR to below control levels. These findings strongly support the view that intrarenal rather than systemic effects of ECF volume expansion are the primary determinants of the resultant natriuresis.

\section{METHODS}

All studies were performed on male Charles River rats (200-250 g, Charles River Breeding Laboratories, Inc., Wilmington, Mass.), fed a standard rat chow diet and allowed water ad lib. The rats were prepared for study as described previously $(5,6)$, with the exception that in the studies in which the aorta was partially clamped, the femoral artery rather than the carotid artery was cannulated for blood pressure measurement and blood sampling. Upon completion of the surgical preparation, all animals were required to meet the following criteria or the experiment was discontinued: blood pressure greater than $95 \mathrm{~mm} \mathrm{Hg}$; temperature $36-39^{\circ} \mathrm{C}$; urine free of blood; and Lissamine green transit time less than $13 \mathrm{~s}$ with dye distributed evenly to all areas of the kidney and with no dye retention in tubules.

If the above conditions were met, $25 \mu \mathrm{Ci}$ of $\left[{ }^{14} \mathrm{C}\right]$ inulin in $0.3 \mathrm{ml}$ of isotonic saline was given as a bolus and an

${ }^{1}$ Abbreviations used in this paper: ECF, extracellular fluid; GFR, glomerular filtration rate. isotonic saline infusion containing $\left[{ }^{14} \mathrm{C}\right]$ inulin in amounts sufficient to deliver $25 \mu \mathrm{Ci} / \mathrm{h}$ was begun at a hydropenic rate of $1.7 \mathrm{ml} / \mathrm{h}$. After a 30 -min equilibration period, the experimental protocols described below were begun.

\section{Experimental protocols}

Three experimental protocols were carried out. In the first protocol, the effect of saline loading on GFR, sodium excretion, and interstitial pressure was studied without aortic clamping ("unclamped studies"). In the remaining two experimental protocols, the effect of a reduction in renal artery pressure on the natriuresis induced by volume expansion was examined. All three protocols consisted of a control period followed by a period of saline loading. In the control period, the sustaining infusion of isotonic saline was continued at $1.7 \mathrm{ml} / \mathrm{h}$. In the saline loading period, the infusion rate of isotonic saline was increased to $24 \mathrm{ml} / \mathrm{h}$ ind was continued at this rate for the remainder of the experiment.

In the two protocols in which renal perfusion pressure was reduced, renal artery pressure was reduced after a 45min period of saline loading had elapsed in one protocol ("delayed clamping" studies), and at the onset of saline loading in the other protocol ("immediate clamping" studies). In both of these protocols femoral artery pressure was reduced to $70-75 \mathrm{~mm} \mathrm{Hg}$ by tightening a suture placed around the aorta above the left renal artery but below the right renal artery. This level of pressure was chosen because in a preliminary series of delayed clamping experiments, it resulted in the desired experimental model, in which GFR was reduced to approximately $70-100 \%$ of the pre-expansion values in the majority of the rats studied. In the delayed clamping experiments, rats were accepted for study only if GFR was reduced below the hydropenic control value during the period of aortic clamping.

During the control period in all experiments, two timed urine samples (15-30 min each) were collected for measurement of sodium excretion and inulin clearance. In the unclamped studies, these measurements were repeated after 45 min of saline loading had elapsed. The timing of the urine collections during the saline loading period in the experiments in which renal perfusion pressure was decreased is described below.

Delayed clamping experiments. $30 \mathrm{~min}$ after the onset of saline loading, a single 15-min urine collection was obtained. Left renal artery pressure was then reduced to 70-75 $\mathrm{mm} \mathrm{Hg}$ as described above, and was maintained at this level for the remainder of the experiment. $15 \mathrm{~min}$ after left renal artery pressure was reduced, two additional urine samples were collected for measurement of sodium excretion and inulin clearance. The period of aortic clamping ranged from 40 to $80 \mathrm{~min}$.

Immediate clamping experiments. In these studies, left renal artery pressure was reduced to $70-75 \mathrm{~mm} \mathrm{Hg}$ simultaneously with an increase in the infusion rate of saline to $24 \mathrm{ml} / \mathrm{h}$, as described above. After $30 \mathrm{~min}$ of saline loading and aortic constriction, two timed urine samples were collected while femoral artery pressure was maintained at 70-75 mm Hg. After completion of these collections, the aortic clamp was released and 15 min later, one further timed urine sample was collected. The period of aortic clamping ranged from 60 to $110 \mathrm{~min}$.

\section{Renal plasma flow measurements}

Renal plasma flow was measured in four rats in the delayed clamping studies and in five rats in the immediate clamping studies. In these studies, in addition to arterial 
TABLE I

Renal Function, Blood Pressure (BP), and Hematocrit before and during Saline Loading in the Three Experimental Groups

\begin{tabular}{|c|c|c|c|c|c|c|}
\hline & $\mathrm{U} / \mathrm{P}$ In & GFR & V/GFR & UNaV* & BP\& & Het \\
\hline & & $m l / m i n$ & $\%$ & $\mu e q / \min$ & $m m \mathrm{Hg}$ & $m l / 100 \mathrm{ml}$ \\
\hline \multicolumn{7}{|c|}{ Unclamped (17 rats) } \\
\hline Control & $545 \pm 54$ & $1.4 \pm 0.1$ & $0.2 \pm 0.02$ & $0.1 \pm 0.01$ & $122 \pm 3$ & $45 \pm 0.7$ \\
\hline Early saline & $27 \pm 6$ & $1.7 \pm 0.1$ & $5.7 \pm 0.7$ & $15.9 \pm 2.0 \ddagger$ & $121 \pm 3$ & $40 \pm 0.7$ \\
\hline Late saline & $19 \pm 3$ & $1.8 \pm 0.1$ & $6.8 \pm 0.8$ & $24.0 \pm 2.8$ & $123 \pm 5$ & $40 \pm 0.5$ \\
\hline \multicolumn{7}{|c|}{ Delayed clamping (11 rats) } \\
\hline Control & $352 \pm 27$ & $1.4 \pm 0.1$ & $0.3 \pm 0.02$ & $0.2 \pm 0.07$ & $112 \pm 3$ & $47 \pm 0.7$ \\
\hline Saline & $40 \pm 5$ & $1.7 \pm 0.1$ & $1.9 \pm 0.4$ & $9.9 \pm 1.1$ & $112 \pm 3$ & $41 \pm 0.9$ \\
\hline Saline + clamp & $165 \pm 36$ & $1.2 \pm 0.1$ & $1.0 \pm 0.3$ & $3.2 \pm 0.9$ & $72 \pm 1$ & $39 \pm 0.8$ \\
\hline \multicolumn{7}{|c|}{ Immediate clamping (11 rats) } \\
\hline Control & $352 \pm 27$ & $1.1 \pm 0.1$ & $0.3 \pm 0.02$ & $0.1 \pm 0.01$ & $119 \pm 3$ & $47 \pm 0.7$ \\
\hline Saline + clamp & $621 \pm 118$ & $1.5 \pm 0.1$ & $0.2 \pm 0.04$ & $0.4 \pm 0.14$ & $73 \pm 1$ & $40 \pm 0.7$ \\
\hline Saline & $21 \pm 2$ & $1.6 \pm 0.1$ & $5.8 \pm 0.9$ & $17.4 \pm 1.9$ & $137 \pm 4$ & $40 \pm 1.0$ \\
\hline
\end{tabular}

Values shown are means \pm SEM.

* Control sodium excretions were measured in eight rats in the unclamped series, and in nine rats in each of the clamped series. $\ddagger$ In the unclamped series sodium excretions during saline loading were measured in 13 rats.

$\S$ In the immediate and delayed clamping experiments, the blood pressure values given are femoral artery pressures.

blood and urine samples, renal venous blood samples were

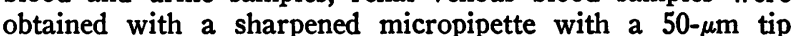
opening, as described by Barratt, Wallin, Rector, and Seldin (7). Renal plasma flow was calculated by the Wolf formula (8), $(U-R V) V /(A-R V)$, where $A, R V$, and $U$ are the activities of $\left[{ }^{14} \mathrm{C}\right]$ inulin in arterial plasma, renal vein plasma, and urine, respectively, while $V$ is the urine flow rate. Filtration fraction was calculated by the formula, $(A-R V) /$ A.

\section{Tubular pressure and diameter measurements}

Proximal and distal tubular pressure and diameter measurements were carried out in all the rats in the unclamped protocol, and in a group of rats from each of the two aortic clamping protocols, to estimate changes in interstitial and transepithelial pressure (see below). In the experiments in which these measurements were made, proximal and distal tubular pressures were measured by a modification of the technique of Gottschalk and Mylle (9) described previously from our laboratory (5), and photographs were taken of the kidney surface after the intravenous injection of Lissamine green for diameter measurements (6). Tubular pressures were measured in at least one proximal and one distal tubule both before and after photographing the kidney surface, and in not less than three proximal and three distal tubules in each experimental period. In the unclamped studies tubular pressure and diameter measurements were made in the control period and repeated after $45 \mathrm{~min}$ of saline loading had elapsed. In both the immediate and delayed clamping experiments, tubular pressures and diameters were measured in the control period and repeated during the period of aortic clamping and saline loading.

Estimation of changes in interstitial pressure and changes in the transepithelial pressure gradient. Changes in interstitial pressure and in the transepithelial pressure gradient during the period of saline loading were estimated from the observed changes in tubular pressure and diameter from the in situ compliance characteristics of the tubules. The definition of tubular compliance and how it is used to estimate changes in transepithelial and peritubular pressures have been described in detail in a previous study from our laboratory (6), and are summarized briefly below.

The in situ compliance characteristics of both the proximal and distal tubule have been defined ( 6 ) by measuring the changes in tubular diameter induced by increasing tubular pressure in individual tubular segments partially obstructed with castor oil. In such a setting, the change in tubular pressure is assumed to be equal to the change in the transepithelial pressure gradient $(\triangle T E P)$, since the peritubular interstitial environment is not perturbed. Proximal tubular compliance was found to be linear over the range of pressures studied, and is described by the equation, $\Delta D=0.5 \times \Delta T E P$, where $\Delta D$ is the change in tubular diameter in microns. Distal tubular compliance was found to be curvilinear and is described by the equation, $\Delta D=$ $15.3-3.3 \times e^{-0.8 \times \triangle T E P+1.5}$.

From these relationships, changes in the transepithelial pressure gradient can be calculated from changes in tubular diameter for the proximal and distal tubule in any setting in which the compliance characteristics of the tubules have not been altered by the experimental maneuver. Changes in interstitial pressure $(\Delta I P)$ are then calculated as the difference between the changes in tubular pressure $(\triangle T P)$ and the change in the transepithelial pressure gradient, $\triangle I P=$ $\Delta T P-\triangle T E P$. Because the distal tubule is markedly compliant (i.e., small changes in the transepithelial pressure produce large changes in diameter), changes in interstitial pressure can be estimated quantitatively by using the distal tubule when tubular pressure is increased by as little as 2 $\mathrm{mm} \mathrm{Hg}$. On the other hand, because the proximal tubule is less compliant, quantitative estimates of interstitial pressure changes can only be carried out with proximal tubular data when proximal tubular pressure is increased by 5 or more $\mathrm{mm} \mathrm{Hg}$ (6). 
TABLE II

Tubular Pressures and Diameters before and during Saline Loading in the

Three Experimental Groups

\begin{tabular}{lccccc}
\hline & \multicolumn{2}{c}{ Proximal tubule } & & \multicolumn{2}{c}{ Distal tubule } \\
\cline { 2 - 3 } \cline { 5 - 6 } & Pressure & Diameter & & Pressure & Diameter \\
\hline Unclamped studies (17) & $m m \mathrm{Hg}$ & $\mu m$ & & $m m \mathrm{Hg}$ & $\mu m$ \\
$\quad$ Control & $11.9 \pm 0.2$ & $15.4 \pm 0.5$ & & $6.8 \pm 0.2$ & $12.9 \pm 0.4$ \\
$\quad$ Saline & $17.1 \pm 0.8$ & $16.9 \pm 0.5$ & & $13.1 \pm 0.9$ & $18.2 \pm 0.7$ \\
Delayed clamping studies (6)* & & & & & \\
$\quad$ Control & $13.2 \pm 0.6$ & $13.3 \pm 0.5$ & & $6.9 \pm 0.5$ & $12.1 \pm 0.3$ \\
$\quad$ Saline + clamp & $13.0 \pm 1.0$ & $12.8 \pm 0.3$ & & $7.1 \pm 0.9$ & $12.3 \pm 0.5$ \\
Immediate clamping studies (4) & & & & & \\
$\quad$ Control & $13.9 \pm 0.5$ & $12.4 \pm 0.6$ & & $7.0 \pm 0.5$ & $12.6 \pm 0.7$ \\
$\quad$ Saline + clamp & $12.7 \pm 0.2$ & $12.0 \pm 0.6$ & & $6.2 \pm 0.2$ & $11.8 \pm 0.5$ \\
\hline
\end{tabular}

Values shown are the means \pm SEM.

* Tubular diameters were measured in four rats in this group.

As noted above, this method of estimating changes in interstitial and transepithelial pressure is based upon the assumption that the experimental agent does not significantly affect the compliance characteristics of the tubule. In the case of isotonic saline loading, such an assumption does not seem unreasonable. As a further check, however, a comparison of the estimates of the change in interstitial pressure obtained independently from the proximal and distal tubules can be carried out. Given the different compliance characteristics of these two segments of the nephron over the range of pressures studied, it would be remarkably fortuitous if a direct effect of saline on tubular compliance yielded similar estimates of the change in interstitial pressure from the proximal and distal tubule.

\section{Analytic methods}

$\left[{ }^{14} \mathrm{C}\right]$ Inulin activity in plasma and urine was measured with a liquid scintillation solution described previously (10). Plasma and urine sodium concentrations were measured with a flame photometer. Tubular diameter measurements were made from photographs of the kidney surface projected on a white cardboard screen by a doubled-blind technique. Only Lissamine green-filled tubular segments were measured, and tubular diameters were arbitrarily taken as the width of the intratubular dye column. The technique of diameter measurement and the controls used were identical to those reported previously (6).

\section{RESULTS}

\section{Acute saline loading without aortic clamping} (17 rats)

The results of these studies are shown in Tables I and II and in Figs. 1 and 2.

GFR and sodium excretion (Fig. 1). The period of saline loading was divided into an early saline period (45-80 min after the onset of saline loading) and a late saline period $(80-120 \mathrm{~min}$ after the onset of saline loading). Mean GFR in the micropunctured kidney increased significantly ${ }^{2}$ from $1.4 \mathrm{ml} / \mathrm{min}$ in the hydropenic control period to $1.7 \mathrm{ml} / \mathrm{min}$ in the early saline period, and increased further to $1.8 \mathrm{ml} / \mathrm{min}$ $(P<0.02)$ in the late saline period. Mean sodium excretion increased from $0.1 \mu \mathrm{eq} / \mathrm{min}$ in the control period to $15.9 \mu \mathrm{eq} / \mathrm{min}$ in the early saline period, and increased further to $24.0 \mu \mathrm{eq} / \mathrm{min}$ in the late saline period.

Tubular pressures and diameters (Table II). In the hydropenic control period, mean proximal tubular pressure was $11.9 \mathrm{~mm} \mathrm{Hg}$, and mean distal tubular pressure was $6.8 \mathrm{~mm} \mathrm{Hg}$. During the period of saline loading, proximal tubular pressure increased significantly to a mean value of $17.1 \mathrm{~mm} \mathrm{Hg}$, and distal tubular pressure increased significantly to a mean value of $13.1 \mathrm{~mm} \mathrm{Hg}$. This increase in tubular pressure occurred within the first $45 \mathrm{~min}$ of saline loading; there was no further significant change in tubular pressure in the late saline period. Proximal tubular diameter increased slightly but significantly from a mean value of $15.4 \mu \mathrm{m}$ in the hydropenic control period to $16.9 \mu \mathrm{m}$ during saline loading, and distal diameter increased significantly from $12.9 \mu \mathrm{m}$ in the control period to 18.2 $\mu \mathrm{m}$ during the period of saline loading.

Estimated changes in interstitial pressure and in the transepithelial pressure gradient (Fig. 2). In 15 out of 17 experiments distal pressure increased by 2 or more $\mathrm{mm} \mathrm{Hg}$ during saline-induced volume expansion. The right-hand panel of Fig. 2 depicts the mean change in interstitial pressure calculated from the observed

${ }^{2}$ Throughout this paper, the term "significant" will indicate a $P$ value of less than 0.01 , unless otherwise indicated. 

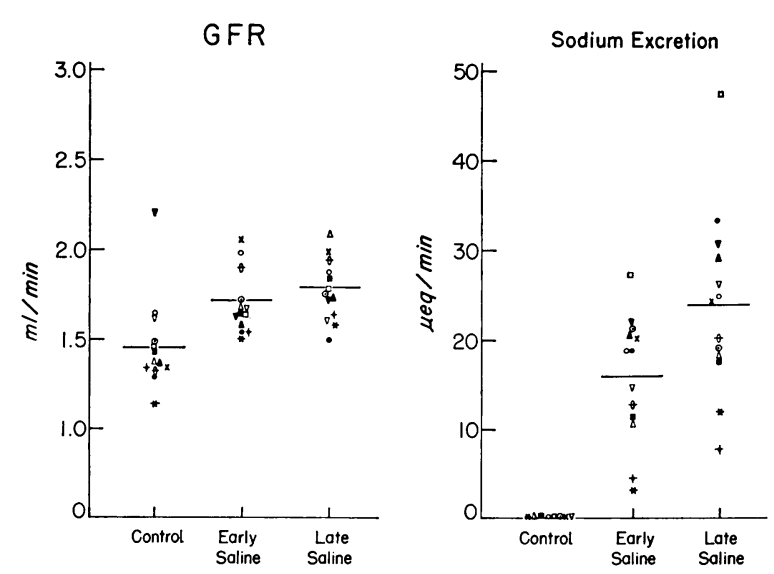

FIGURE 1 GFR and sodium excretion during hydropenia (control) and during saline-induced volume expansion without aortic constriction (unclamped studies). For each animal, the results are obtained from the left kidney and are represented by the same symbol during the control period, 45-80 min after the onset of saline loading (early saline), and $80-120 \mathrm{~min}$ after the onset of saline loading (late saline). Control sodium excretions were measured in only 9 of the 13 rats.

changes in tubular pressure and diameter in these experiments (see Methods). As can be seen, saline loading resulted in a significant increase in interstitial pressure (mean increase $=5.3 \mathrm{~mm} \mathrm{Hg}$ ). In 10 of the 17 experiments, proximal tubular pressure also increased by 5 or more $\mathrm{mm} \mathrm{Hg}$, allowing an independent estimate of the increase in interstitial pressure. On the basis of the changes in proximal tubular pressure and diameter in these 10 experiments, interstitial pressure increased by $4.3 \mathrm{~mm} \mathrm{Hg}$, a value not significantly different from the distal tubular estimate. The finding of closely similar values for the increase in interstitial
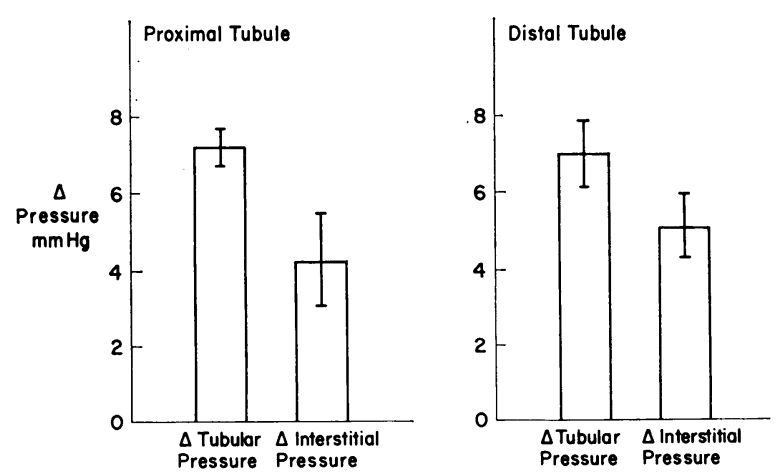

Figure 2 Changes in tubular pressure, and changes in interstitial pressure estimated from proximal tubular pressure and diameter changes (left-hand panel, $n=10$ ), and estimated from distal tubular pressure and diameter changes (right-hand panel, $n=15$ ). In both panels the bars represent the mean change in $\mathrm{mm} \mathrm{Hg} \pm \mathrm{SEM}$.
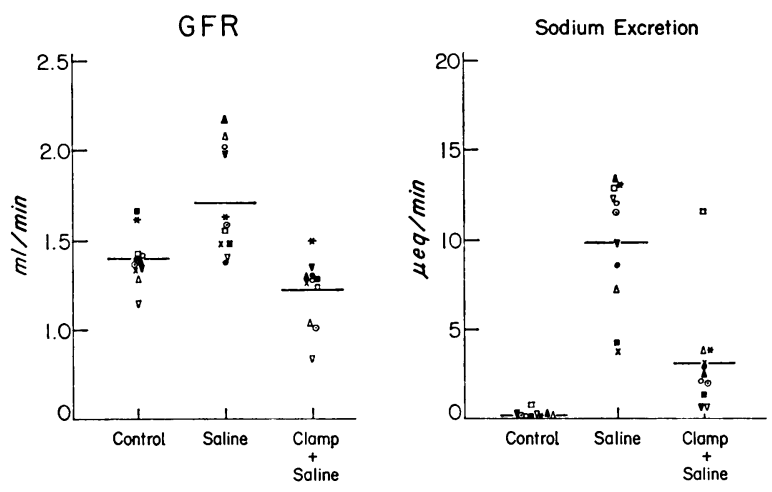

FIGURE 3 GFR and sodium excretion during hydropenia (control), during saline-induced volume expansion (saline), and during continued volume expansion with renal perfusion pressure reduced to between 70 and $75 \mathrm{~mm} \mathrm{Hg}$ in the left kidney (clamp + saline) in the delayed clamping studies. For each animal the results are obtained from the left kidney, and are represented by the same symbol in the three experimental periods. The observations during the saline period were obtained between 30 and $45 \mathrm{~min}$ after the onset of saline loading, and during the clamp + saline period were obtained $15 \mathrm{~min}$ after the reduction in renal perfusion pressure. Control sodium excretions were measured in only 9 of the 11 rats.

pressure calculated independently from the proximal and distal tubules, despite their markedly different compliance characteristics (see Methods), strongly supports the assumption that saline loading has no direct effect on tubular compliance.

Both proximal and distal tubular pressure increased to a greater extent than interstitial pressure during saline loading in these experiments (Fig. 2), and therefore, the transepithelial hydrostatic pressure gradient increased significantly in both segments of the nephron.

Acute saline loading with delayed aortic clamping (11 rats)

The results of these studies are presented in Tables $I$ and II, and in Figs. 3 and 4.

GFR and sodium excretion (Fig. 3). Mean GFR in the micropunctured kidney increased significantly from $1.4 \mathrm{ml} / \mathrm{min}$ in the hydropenic control period to $1.7 \mathrm{ml}$ / $\mathrm{min}$ in the period of saline loading before aortic clamping, and sodium excretion increased from 0.2 to 9.9 $\mu \mathrm{eq} / \mathrm{min}$. Aortic clamping reduced mean GFR to 1.2 $\mathrm{ml} / \mathrm{min}$, a value $91 \%$ of the mean GFR in the control hydropenic period. As expected, despite the reduction in renal perfusion pressure and GFR, sodium excretion remained significantly increased, at $3.2 \mu \mathrm{eq} / \mathrm{min}$.

Tubular pressure and diameter (Table II). Tubular pressure measurements were carried out in six rats, and diameter measurements in four rats in this group. During the period of saline loading and aortic clamping, mean proximal and distal pressure, 13.0 and 7.1 $\mathrm{mm} \mathrm{Hg}$, respectively, were not significantly different 
from control. Proximal and distal tubular diameters during the period of saline loading and aortic clamping, 12.8 and $12.3 \mu \mathrm{m}$, respectively, were also not significantly different from control.

Renal plasma flow and filtration fraction (Fig. 4). Renal plasma flow and filtration fraction were measured in four rats in the delayed clamping group. Renal plasma flow increased from $6.5 \mathrm{ml} / \mathrm{min}$ in the hydropenic control period to $8.9 \mathrm{ml} / \mathrm{min}$ during the period of saline loading, and filtration fraction decreased in three of the four rats. After aortic clamping, renal plasma flow decreased to a mean value of $5.3 \mathrm{ml} / \mathrm{min}$, and filtration fraction increased to 0.27 ; these values were not significantly different from control.

Acute saline loading with immediate aortic clamping (11 rats)

The results of these studies are presented in Tables I and II and in Figs. 4 and 5.

GFR and sodium excretion (Fig. 5). Mean GFR in the micropunctured kidney increased from $1.1 \mathrm{ml} /$ $\mathrm{min}$ in the hydropenic control period to $1.5 \mathrm{ml} / \mathrm{min}$ $(P<0.05)$ in the period of saline loading and aortic

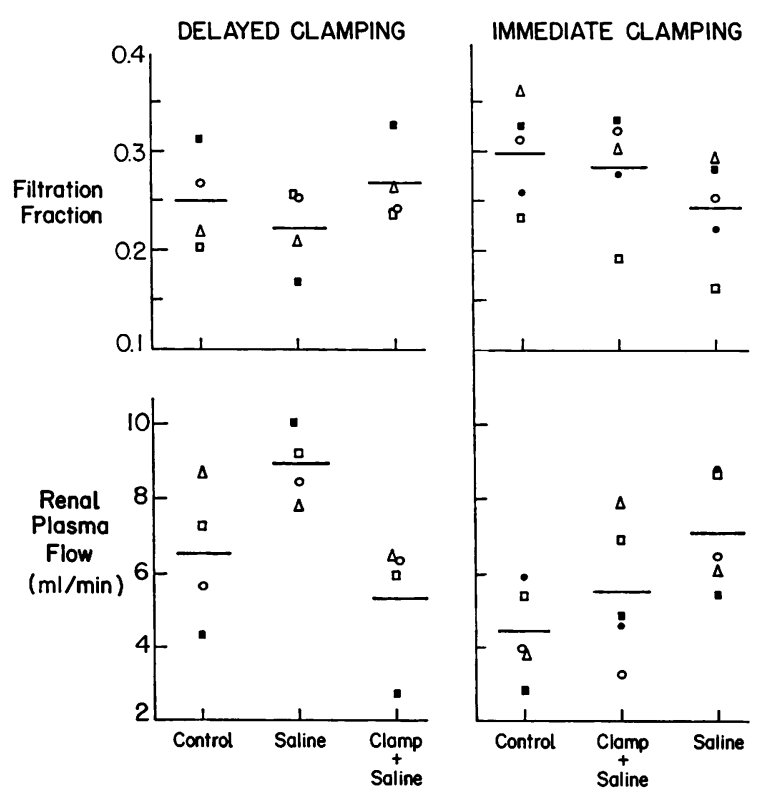

FIGURE 4 Filtration fraction and renal plasma flow in the delayed clamping studies (left-hand panels), and in the immediate clamping studies (right-hand panels). For each animal, the results are obtained from the left kidney, and are represented by the same symbol in the control period, the period of saline loading without aortic constriction (saline), and the period of saline loading with renal perfusion pressure reduced to $70-75 \mathrm{~mm} \mathrm{Hg}$ (clamp + saline).
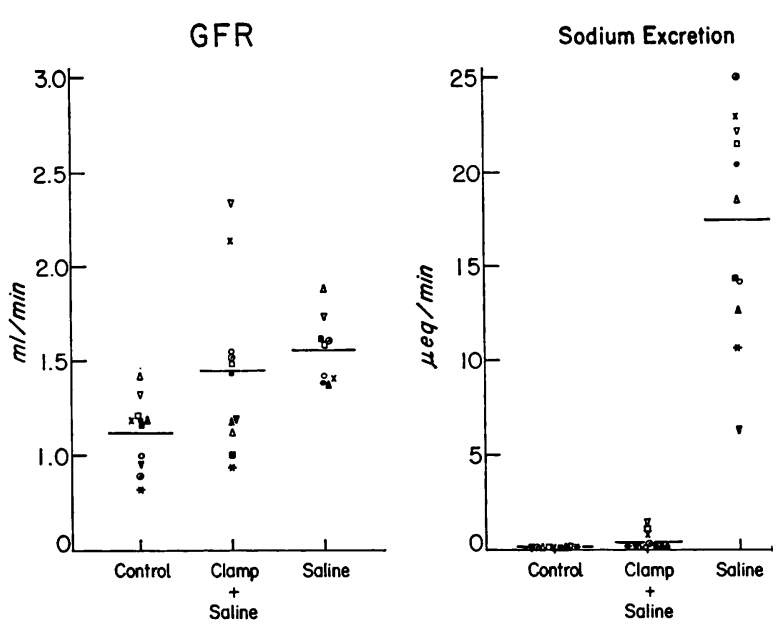

FIGURE 5 GFR and sodium excretion during hydropenia (control), during saline-induced volume expansion with renal perfusion pressure reduced to between 70 and $75 \mathrm{~mm}$ $\mathrm{Hg}$ at the onset of saline loading (clamp + saline), and during continued volume expansion without control of renal perfusion pressure (saline) in the immediate clamping studies. For each animal, the results are obtained from the left kidney and are represented by the same symbol during the three experimental periods. The observations during the clamp + saline period were obtained between 30 and 110 min of saline loading, and in the saline period were obtained $15 \mathrm{~min}$ after release of the aortic clamp. Control sodium excretions were measured in only 9 of the 11 rats, and GFR was measured in only 9 of the 11 rats during the saline period.

constriction. ${ }^{3}$ Despite the resultant increase in the filtered load of sodium, mean sodium excretion increased only trivially, from $0.1 \mu \mathrm{eq} / \mathrm{min}$ to $0.4 \mu \mathrm{eq} / \mathrm{min}(P<0.05)$. This value was significantly lower than the mean sodium excretion during the period of aortic constriction in the delayed clamping studies $(3.2 \mu \mathrm{eq} / \mathrm{min})$. When the aortic clamp was released, mean GFR did not change significantly, increasing in five rats, and decreasing in four rats. However, urinary sodium excretion increased dramatically in all animals to a mean value of $17.4 \mu \mathrm{eq} / \mathrm{min}$.

In two rats in this group both ureters were catheterized so that urinary sodium excretion could be measured from the unclamped as well as from the clamped kidney. In these two studies, sodium excretions from the contralateral unclamped kidney were 9.4 and 12.0 $\mu \mathrm{eq} / \mathrm{min}$, respectively, during the period of saline loading, as compared to 0.2 and $0.01 \mu \mathrm{eq} / \mathrm{min}$ on the clamped side.

${ }^{3}$ Although these results are based upon GFR measurements begun after $30 \mathrm{~min}$ of aortic constriction and saline loading, it should be noted that a similar pattern is present in the period immediately after aortic constriction. In three rats in which GFR was measured during the first 20 min of aortic constriction and saline loading, GFR increased in two (by 8 and 13\%) and decreased in one (by 16\%). 
Tubular pressures and diameters (Table II). Tubular pressure and diameter measurements were carried out in four rats in this group. Mean proximal and distal pressures during the period of aortic clamping and saline loading, 12.7 and $6.2 \mathrm{~mm} \mathrm{Hg}$, respectively, were not signiticantly different from control. Similarly, proximal and distal tubular diameters during the period of aortic clamping and saline loading, 12.0 and $11.8 \mu \mathrm{m}$, respectively, were not significantly different from control.

Renal plasma flow and filtration fraction (Fig. 4). Renal plasma flow and filtration fraction were measured in five rats in the immediate clamping group. During the period of aortic clamping, renal plasma flow $(5.5 \mathrm{ml} / \mathrm{min})$ and filtration fraction (0.28) were not significantly different from the hydropenic control values $(4.4 \mathrm{ml} / \mathrm{min}$ and 0.30$)$. With release of the aortic clamp, renal plasma flow increased to $7.1 \mathrm{ml} /$ min and filtration fraction fell to $0.24(P<0.05)$.

\section{DISCUSSION}

The present studies have demonstrated that the natriuretic response to ECF volume expansion is markedly different when renal perfusion pressure is reduced at the onset of saline loading rather than after a period of saline loading has elapsed. As shown in Fig. 5, sodium excretion increased only trivially, despite massive ECF volume expansion and an increase in GFR when renal perfusion pressure was promptly reduced to $70 \mathrm{~mm} \mathrm{Hg}$ at the onset of saline loading. By contrast, when renal perfusion pressure was reduced to an identical level after $45 \mathrm{~min}$ of saline loading, sodium excretion remained markedly increased, even though GFR was below pre-expansion levels.

These findings argue strongly that neither a circulating hormone nor a reduction in plasma oncotic pressure is responsible for the diuresis induced by volume expansion. If a hormone were responsible for the persistent diuresis in the delayed clamping experiments, it should also have produced a diuresis in the immediate clamping experiments, since these rats were massively volume expanded and since the right unclamped kidney was having the expected diuretic response. Similarly, these findings provide little support for the hypothesis that a reduction in systemic plasma oncotic pressure is the critical determinant of the diuresis induced by volume expansion. Since the vascular compartment was expanded during saline loading to a virtually identical degree in both the immediate and delayed clamping studies (38 and $41 \%$, respectively, on the basis of the fall in hematocrit), it is reasonable to infer that plasma oncotic pressure was reduced to the same degree in both settings. Furthermore, in the absence of any further alteration in plasma volume (or
GFR), release of the aortic clamp in the immediate clamping experiments led to a marked increase in sodium excretion (from 0.4 to $17.4 \mu \mathrm{eq} / \mathrm{min}$ ).

The difference in response between the immediate and delayed clamping experiments indicates that intrarenal rather than systemic factors are the critical determinants of the natriuretic response to ECF volume expansion. In addition, these experiments provide a tool for examining what factors within the kidney might be playing a role in producing the diuresis. In the present study, we have used this difference in response to evaluate whether changes in interstitial hydrostatic pressure (defined as the pressure opposing tubular dilatation) might be playing a major role in producing the natriuresis. As shown in Fig. 2, saline loading in the absence of aortic clamping results in a 4-5 $\mathrm{mm} \mathrm{Hg}$ increase in interstitial pressure, as estimated both from the proximal and distal tubule. The finding of an increase in interstitial hydrostatic pressure in these studies is in agreement with measurements of subcapsular pressure in the rat (11) and in the $\operatorname{dog}$ (12) after acute saline infusions. The increase in interstitial pressure during saline loading has been suggested by Lewy and Windhager (13) and others $(14,15)$ to be the factor that ultimately produces the reduction in proximal sodium reabsorption.

The results of the studies in which renal perfusion pressure was reduced, however, provide little support for the view that a sustained large increase in interstitial hydrostatic pressure plays any important role in the natriuretic response to saline loading. When renal artery pressure was reduced after an interval of saline loading had elapsed, a setting in which proximal sodium reabsorption is depressed (2) and sodium excretion increased (Fig. 3), tubular pressures and diameters were virtually identical to their respective control values, indicating that interstitial pressure had returned to the hydropenic control value. Although it is conceivable that there might still be a small increase in interstitial pressure, i.e., less than $2 \mathrm{~mm} \mathrm{Hg}$ (which would not be detectable by this technique) during aortic clamping in these studies, it is clear that the large increase in interstitial pressure found in the unclamped studies is no longer present.

In addition, the results of the aortic clamping studies provide little support for the view that sustained alterations in renal plasma flow and filtration fraction are crucial determinants of the natriuresis. In both the immediate and delayed clamping experiments, renal plasma flow and filtration fraction were indistinguishable from the control hydropenic values during the period of reduced renal artery pressure and saline loading. 
Although sustained alterations in renal plasma flow and interstitial hydrostatic pressure cannot be invoked as determinants of the natriuretic response to ECF volume expansion, it should be noted that in all experiments in which a natriuresis occurred, the kidney was in fact exposed to at least a transient increase in both parameters. Both in the unclamped experiments and in the delayed clamping experiments before aortic clamping, interstitial pressure and renal plasma flow were increased. In contrast, in the immediate clamping experiments, in which no natriuresis occurred during saline loading, the kidney was not exposed even transiently to an increase in either interstitial pressure or renal plasma flow. These studies strongly suggest, therefore, that ECF volume expansion can induce a natriuresis only if the kidney has been exposed to at least a transient increase in interstitial pressure or renal plasma flow (or both). In addition, this transient exposure must produce sustained changes in the peritubular environment that reduce sodium reabsorption, since a subsequent reduction in renal artery pressure does not eliminate the diuresis.

The natriuresis induced by volume expansion has been well documented to result from a combination of increased delivery of sodium out of the proximal tubule and an impairment of the ability of the more distal portions of the nephron to recapture the additional sodium delivered from the proximal tubule $(16,17)$. Since impairment of sodium reabsorption in both the proximal and distal nephron appears to be required for the natriuresis to occur (16), the increase in interstitial hydrostatic pressure and renal plasma flow that occurs when the kidney is exposed to volume expansion at a normal perfusion pressure could have its predominant effect on sodium reabsorption at either site in the nephron, or at both sites. In the succeeding discussion, we will first examine the possible mechanisms by which an increase in hydrostatic pressure and renal plasma flow can produce sustained alterations in the peritubular environment of the proximal tubule, and then we will examine the possible effects of these two factors on the peritubular environment in the more distal portions of the nephron.

The changes in the transepithelial hydrostatic pressure gradient during saline loading described in the present experiments, in association with the recent measurement of subcapsular fluid protein concentration by Wolgast, Persson, Schnermann, Ulfendahl, and Wunderlich (18), allow a critical evaluation of the hypothesis that the observed reduction in proximal sodium reabsorption is the result of a reduction in transepithelial Starling forces. In Fig. 6, we have used these data to depict schematically the net transepithelial hydrostatic and oncotic pressure gradients during hydro-
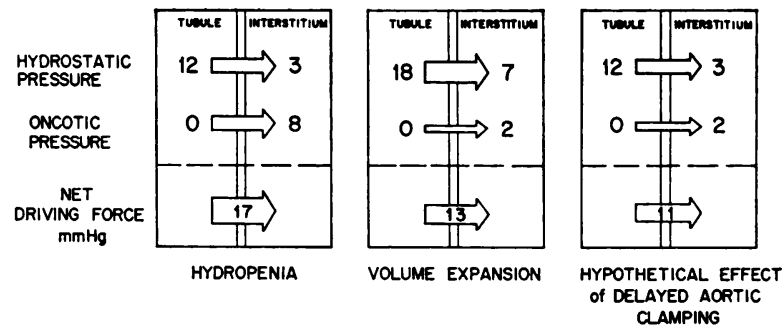

FIGURE 6 Schematic representation of the net Starling forces favoring fluid movement from the tubule to the peritubular interstitium during hydropenia (left-hand panel) and during saline-induced volume expansion (center panel). The right-hand panel depicts a hypothetical model of the net Starling forces when renal perfusion pressure is reduced after a period of saline loading has elapsed.

penia and during saline loading. From this representation of the data it is apparent that the net transepithelial hydrostatic pressure gradient is increased rather than decreased during saline loading, since tubular pressure increases more than interstitial pressure." These data indicate, therefore, that if a reduction in the transepithelial Starling forces is the factor that produces the depression in proximal sodium reabsorption during saline loading, then interstitial fluid oncotic pressure must be decreased. As shown in Fig. 6, measurements of subcapsular fluid protein concentration during hydropenia and during saline loading are in fact consistent with a fall in interstitial oncotic pressure of approximately $6 \mathrm{~mm} \mathrm{Hg}$.

During aortic clamping in both the immediate and delayed clamping experiments, the transepithelial hydrostatic pressure gradient was not significantly changed from control. To account for the difference in sodium excretion in these two settings on the basis of differences in transepithelial Starling forces, therefore, one must postulate that interstitial fluid oncotic pressure is reduced in the delayed clamping experiments (as shown in the right-hand panel of Fig. 6) but not in the immediate clamping experiments. Since plasma oncotic pressure is reduced to the same degree in both settings, however, a difference in interstitial fluid oncotic pressure would require that some mechanism other than a reduction in peritubular capillary protein concentration is responsible for inducing changes in interstitial fluid oncotic pressure. The transient increase

\footnotetext{
'Although Daugharty, Ueki, Nicholas, and Brenner (2) have recently reported that proximal tubular pressure is not increased in volume-expanded rats, it should be noted that in their studies, tubular pressure was measured only after the volume-expanding stimulus had been discontinued. Proximal tubular pressure must have been increased in their experiments during the induction of volume expansion, however, since the rate of infusion was identical to that used in our studies.
} 
in interstitial pressure (and renal plasma flow) observed in the present experiments might conceivably be the factor necessary to induce this critical alteration in interstitial fluid composition. The hypothesis that differences in interstitial fluid oncotic pressure (and therefore in proximal tubular sodium reabsorption) are responsible for the difference in sodium excretion in the immediate and delayed clamping experiments, of course, remains speculative, but is readily amenable to experimental testing.

Alternatively, it is possible that proximal tubular sodium reabsorption is depressed in both the immediate and delayed clamping experiments, but that the capacity of the distal nephron to recapture the increased sodium delivery is impaired only in the delayed clamping experiments. One mechanism by which an increase in interstitial pressure and renal plasma flow might impair the capacity of the loop of Henle and collecting duct to increase sodium reabsorption is by reducing medullary interstitial solute content (4).

The present experiments cannot, of course, exclude the possibility that intrarenal effects other than those measured might play a major role in producing the natriuresis. Further studies will be required to determine the precise mechanism responsible for the natriuresis and the site within the nephron where this effect occurs. Regardless of the mechanism, however, it is apparent from these studies that a period of exposure of the kidney to the effects of saline loading at a normal perfusion pressure profoundly alters its response to a subsequent reduction in pressure. The absence of a diuretic response despite massive loading in the immediate clamping experiments, in which the kidney was not exposed to these effects, indicates that intrarenal factors are the critical determinants of the natriuretic response to saline loading.

\section{ACKNOWLEDGMENTS}

We are indebted to Dr. Jordan J. Cohen for his constructive criticisms of the manuscript.

This study was supported in part by grants. (HL-5309 and HL-759) from the Heart and Lung Institute and grant (AM-15889) from the Arthritis and Metabolism Institute of the National Institutes of Health.

\section{REFERENCES}

1. deWardener, H. E., I. H. Mills; W. F. Clapham, and C. J. Hayter. 1961. Studies on the efferent mechanism of the sodium diuresis which follows the administration of intravenous saline in the dog. Clin. Sci. (Oxf.). 21: 249-258.

2. Daugharty, T. M., I. F. Ueki, D. P. Nicholas, and B. M. Brenner. 1972. Comparative renal effects of ison- cotic and colloid-free volume expansion in the rat. $\mathrm{Am}$. J. Physiol. 222 : 225-235.

3. Levinsky, N. G., and R. C. Lalone. 1963. The mechanism of sodium diuresis after saline infusion in the dog. J. Clin. Invest. 42: 1261-1276.

4. Earley, L. E., and R. M. Friedler. 1965. Changes in renal blood flow and possibly the intrarenal distribution of blood during the natriuresis accompanying saline loading in the dog. J. Clin. Invest. 44: 929-941.

5. Cortell, S., M. Davidman, F. J. Gennari, and W. B. Schwartz. 1972. Catheter size as a determinant of outflow resistance and intrarenal pressure. $\mathrm{Am}$. J. Physiol. 223: 910-915.

6. Cortell, S., F. J. Gennari, M. Davidman, W. H. Bossert, and W. B. Schwartz. 1973. A definition of proxi$\mathrm{mal}$ and distal tubular compliance. J. Clin. Invest. 52: 2330-2339.

7. Barratt, L. J., J. D. Wallin, F. C. Rector, Jr., and D. W. Seldin. 1973. Influence of volume expansion on single-nephron filtration rate and plasma flow in the rat. Am. J. Physiol. 224 : 643-650.

8. Pitts, R. F. 1968. Physiology of the Kidney and Body Fluids. Year Book Medical Publishers, Inc., Chicago, Ill., 2nd edition. 145.

9. Gottschalk, C. W., and M. Mylle. 1956. Micropuncture study of pressures in proximal tubules and peritubular capillaries of the rat kidney and their relation to ureteral and renal venous pressure. Am. J. Physiol. 185: 430-439.

10. Gennari, F. J., S. Cortell, and W. B. Schwartz. 1970 Loss of measured activity of inulin- ${ }^{16} \mathrm{C}$ and a corrective technique. J. Appl. Physiol. 28: 105-107.

11. Schnermann, J. E., H. Persson, L. Ulfendahl, M. Wolgast, and P. Wunderlich. 1972. Functional characteristics of the renal interstitial space. In Recent Advances in Renal Physiology. H. Wirz and F. Spinelli, editors. S. Karger AG., Basel 43-50.

12. Hebert, L. A., and G. S. Arbus. 1971. Renal subcapsular pressure-a new intrarenal pressure measurement. Am. J. Physiol. 220: 1129-1136.

13. Lewy, J. E., and E. E. Windhager. 1968. Peritubular control of proximal tubular fluid reabsorption in the rat kidney. Am. J. Physiol. 214: 943-954.

14. Martino, J. A., and L. E. Earley. 1967. Demonstration of a role of physical factors as determinants of the natriuretic response to volume expansion. J. Clin. In vest. 46: 1963-1978.

15. Bculpaep, E. L. 1972. Permeability changes of the proximal tubule of necturus during saline loading. Am J. Physiol. 222 : 517-531.

16. Stein, J. H., R. W. Osgood, S. Boonjarern, and T. F. Ferris. 1973. A comparison of the segmental analysis of sodium reabsorption during Ringer's and hyperoncotic albumin infusion in the rat. J. Clin. Invest. 52: 2313-2323.

17. Bennett, C. M. 1973. Effect of extracellular volume expansion upon sodium reabsorption in the distal nephron of dogs. J. Clin. Invest. 52: 2548-2555.

18. Wolgast, M., E. Persson, J. Schnermann, H. Ulfendahl, and P. Wunderlich. 1973. Colloid osmotic pressure of the subcapsular interstitial fluid of rat kidneys during hydropenia and volume expansion. Pflügers Arch. Eur. J. Physiol. 340: 123-131. 